\title{
Anodic-Oxide-Induced Intermixing in GaAs-AlGaAs Quantum-Well and Quantum-Wire Structures
}

\author{
Shu Yuan, Member, IEEE, Chennupati Jagadish, Senior Member, IEEE, Yong Kim, Yong Chang, \\ Hark Hoe Tan, Member, IEEE, Richard M. Cohen, Mladen Petravic, Lap Van Dao, \\ Mike Gal, Senior Member, IEEE, Michael C. Y. Chan, E. Herbert Li, Senior Member, IEEE, \\ Jeong-seok O, and Peter S. Zory, Jr., Fellow, IEEE
}

(Invited Paper)

\begin{abstract}
Anodic oxides of GaAs were shown to enhance the intermixing in GaAs-AlGaAs quantum wells $(\mathrm{QW})$ during rapid thermal processing. Proximity of the anodic oxide to the $Q W$ has been shown to influence the photoluminescence (PL) energy shift due to intermixing. Anodic oxide induced intermixing has been used to enhance quantum-wire PL in the structures grown on V-groove patterned GaAs substrates. This has been attributed to enhanced lateral confinement in these structures. Injection of defects such as group-III vacancies or interstitials was considered to be driving force for the intermixing.
\end{abstract}

Index Terms - Diffusion processes, oxidation, quantum wells, quantum wires, rapid thermal processes.

\section{INTRODUCTION}

Q UANTUM-WELL intermixing (QWI) (interdiffusion) is very promising for postgrowth modification of materials properties and for fabrication of optoelectronic integrated circuits [1]-[4]. There are currently two kinds of interdiffusion techniques, one is the impurity-induced interdiffusion (IID) [5]-[7], another is the impurity-free interdiffusion (IFID) [3], [8]. IID usually introduces substantial undesired changes in the material resistivity and trap concentrations. IFID appears to be able to generate large bandgap energy shifts without such problems associated with IID. Usually,

Manuscript received January 19, 1998; revised April 28, 1998. This work was supported in part by the Australian Research Council, in part by the Research Grants Council of Hong Kong, in part by the Australian Department of Industry, Science and Tourism, and in part by the Australian Agency for International Development (AusAID) through IDP Education Australia under the Australia-China Institutional Links Program.

S. Yuan, C. Jagadish, H. H. Tan, and M. Petravic are with Department of Electronic Materials Engineering, Research School of Physical Sciences and Engineering, Australian National University, Canberra, ACT 0200, Australia.

Y. Kim is with Korea Institute of Science and Technology, Cheongryang, Seoul 130-650, Korea.

Y. Chang is with Shanghai Institute of Technical Physics, Shanghai, China

R. Cohen is with Department of Materials Science and Engineering, University of Utah, Salt Lake City, UT 84112 USA.

L. V. Dao and M. Gal are with School of Physics, University of New South Wales, Sydney, NSW 2052, Australia.

M. C. Y. Chan and E. H. Li are with Department of Electrical and Electronic Engineering, University of Hong Kong, Pokfulam Road, Hong Kong.

J. O and P. S. Zory, Jr. are with Department of Electrical Engineering, University of Florida, Gainsville, FL 32611 USA.

Publisher Item Identifier S 1077-260X(98)05854-7. a dielectric layer is deposited on the surface of a quantumwell (QW) structure and the sample is annealed at elevated temperature. Depending on the dielectric material used, the encapsulant dielectric layer can enhance or suppress intermixing of the QW structure [9]. The dielectric layer can be deposited using chemical vapor deposition, electron-beam evaporation, or by other means [8]. In GaAs-AlGaAs system, $\mathrm{SiO}_{2}$ and $\mathrm{Si}_{3} \mathrm{~N}_{4}$ are commonly used to promote and reduce interdiffusion, respectively. $\mathrm{SiO}_{2}$, however, reacts with $\mathrm{Al}$ when in direct contact with $\mathrm{AlGaAs}$, and thus generates $\mathrm{Si}$ which behaves as an impurity source. $\mathrm{Si}_{3} \mathrm{~N}_{4}$, on the other hand, causes considerable strain [3]. Alternatively, anodic oxide can be used as the encapsulant layer. Initial study showed that anodic oxide significantly increased the intermixing in GaAs-AlGaAs QW structures [10]. Anodic oxidization is an early way of making native oxide [11]. The pulsed anodization technique has recently attracted attention as a new way of forming current blocking layers for ridge-waveguide QW laser fabrication, since it was simple, reliable, and cost-effective [12]-[13]. We have recently applied this technique to enhance the photoluminescence (PL) and cathodoluminescence signals from V-grooved quantum wires and obtained both spectrally and spatially well-resolved light emission from quantum wires [14]. Like other intermixing techniques, this interdiffusion technique can be conveniently applied to the fabrication of multiple wavelength lasers and nonabsorbing mirrors for highpower laser applications.

In this paper, we review the effect of pulsed anodic oxide on the intermixing in GaAs-AlGaAs quantum structures. The experimental details will be given in Section II, and the results and discussions will be presented in Section III. The paper is summarized in Section IV.

\section{EXPERIMENTAL DETAILS}

The sample structures used for this work were grown by low pressure metal-organic chemical vapor deposition (MOCVD) on $\mathrm{p}^{+} \mathrm{GaAs}$ substrates. The substrates were $\mathrm{Zn}$-doped to a concentration of $1 \times 10^{19} \mathrm{~cm}^{-3}$ and were of $2^{\circ}$ off $\langle 100\rangle$ toward $\langle 110\rangle$. A GaAs buffer layer was grown on the substrate, 


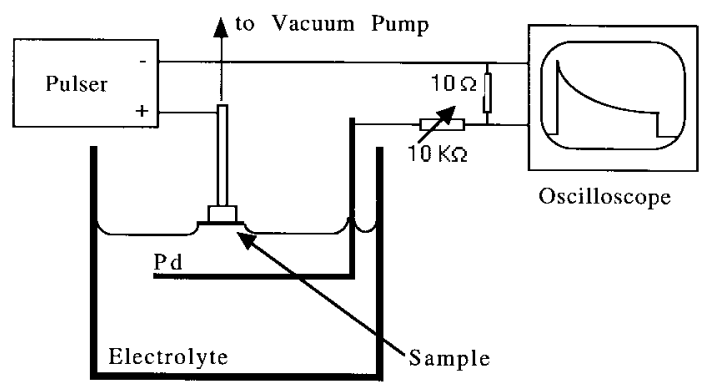

(a)

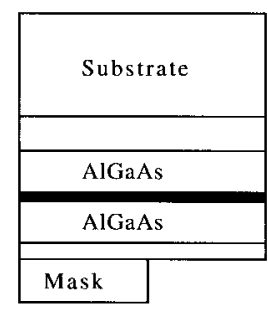

(b)

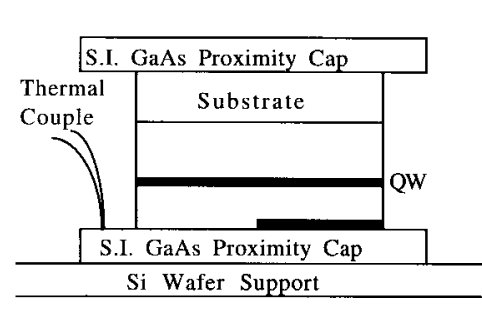

(d)

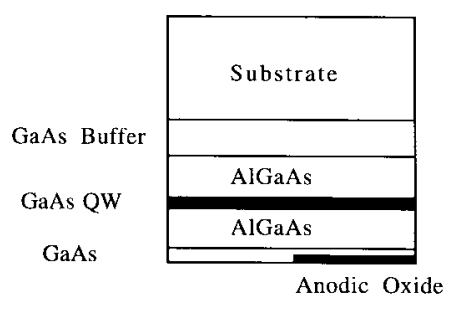

(c)

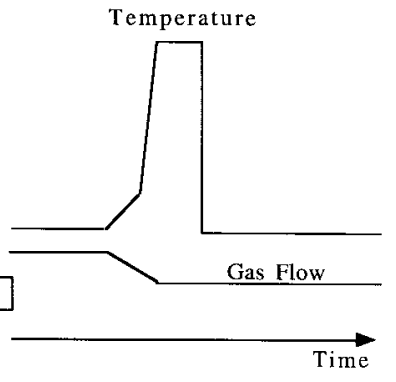

(e)
Fig. 1. (a) Experimental setup for pulsed anodic oxidization. The electrolyte was a solution of ethylene glycol:phosphoric acid:de-ionized water (40:20:1 by volume). The sample was held by the vacuum tweezer. The variable resistor was used to adjust the current density at the sample surface. The current was monitored with the oscilloscope. (b) Typical single QW sample with a mask on it to protect half of the surface before anodization. (c) Sample after anodization and removal of the mask. Half of the surface was converted into anodic oxide. (d) Sample load in the rapid thermal process chamber. Heating was provided by banks of lamp. (e) Typical programmed temperature and $\mathrm{Ar}$ gas flow.

and the thickness was between 100 and $1000 \mathrm{~nm}$ for different samples. For all the samples, the top layer was GaAs, the barrier layers were $\mathrm{AlGaAs}$, and the QW layers were GaAs. All epilayers were nominally undoped and residual doping concentration was $\sim 10^{15} \mathrm{~cm}^{-3}$.

The pulsed anodization was carried out at room temperature [15]. Fig. 1(a) schematically shows the anodization setup. For each sample, half of the surface was covered with thermal glue. A vacuum tube was used as the vacuum tweezer to hold the sample and conduct current. The electrolyte was made of ethylene glycol:phosphoric acid:de-ionized water $(40: 20: 1$ by volume). The voltage as measured across the two output terminals on the pulser was $50 \mathrm{~V}$. The initial current was set according to the current density at the sample surface. The current density used in this paper was in the range from 40 to $160 \mathrm{~mA} / \mathrm{cm}^{2}$. The pulsewidth was $1 \mathrm{~ms}$, while the pulse period was $12 \mathrm{~ms}$. The total anodization time was fixed at $4 \mathrm{~min}$. Under such conditions, the final anodic oxide thickness was

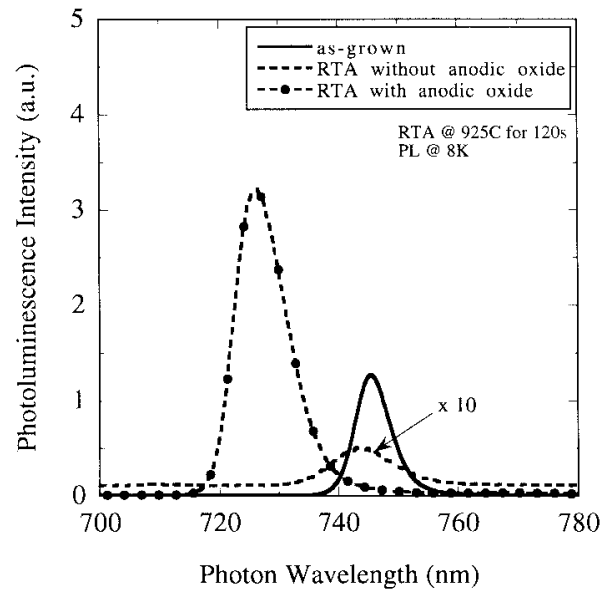

Fig. 2. PL spectra of a single $\mathrm{GaAs}-\mathrm{Al}_{0.3} \mathrm{Ga} 0.7 \mathrm{As} \mathrm{QW}$. The $\mathrm{QW}$ was $3 \mathrm{~nm}$ thick, and the barriers were $50 \mathrm{~nm}$ thick. The solid line was from an as-grown sample. Another sample was annealed at $925^{\circ} \mathrm{C}$ for $120 \mathrm{~s}$ with half area anodized (dashed-dotted line) and half area unanodized (dashed line). The anodization was carried out at $120 \mathrm{~mA} / \mathrm{cm}^{2}$.

in the range of 60-140 $\mathrm{nm}$. Using higher voltage output from the pulser, higher current density, or higher pulse duty cycle, thicker anodic oxide can be obtained [16]. The area covered with thermal glue was unanodized, while anodization took place in the uncovered area to form an oxide layer, this is illustrated in Fig. 1(b). After anodization, samples were rinsed with de-ionized water, followed by acetone to remove the thermal glue, then by de-ionized water and $\mathrm{N}_{2}$ blow dried [Fig. 1(c)]

The intermixing was carried out in a rapid thermal annealing (RTA) chamber with GaAs proximity caps in flowing argon ambient, as shown schematically in Fig. 1(d). The temperature was first slowly ramped up to $100{ }^{\circ} \mathrm{C}$ from room temperature (RT) in $1 \mathrm{~min}$, and then was increased rapidly to the desired temperature (about $900{ }^{\circ} \mathrm{C}$ ) at a ramp rate of $50{ }^{\circ} \mathrm{C} / \mathrm{s}$. After RTA, it cools down quickly [Fig. 1(e)]. PL measurements were performed at $8 \mathrm{~K}$ using an Ar-ion laser beam (514.5 nm), a spectrometer, and a Si CCD camera. Cares were taken to ensure the PL collection efficiency was about the same for each sample. For each sample, PL was measured both from the anodized area and unanodized area.

\section{RESUlTS AND DisCUSSIONS}

We first study the effect of anodic oxide on the intermixing in a GaAs-AlGaAs single QW sample. The sample consisted of a 3-nm GaAs sandwiched between two 50-nmthick $\mathrm{Al}_{0.3} \mathrm{Ga}_{0.7} \mathrm{As}$ barrier layers. The top GaAs layer was 300 $\mathrm{nm}$, while the GaAs buffer layer was $1000 \mathrm{~nm}$. Fig. 2 compares the PL spectra from an as-grown sample, the anodized and unanodized areas of another sample. The sample was anodized at $120 \mathrm{~mA} / \mathrm{cm}^{2}$ and the thickness of anodic oxide was about $120 \mathrm{~nm}$. Sample was annealed at $925^{\circ} \mathrm{C}$ for $120 \mathrm{~s}$. Relative to the as-grown sample, the unanodized area showed a marginal blueshift $(5 \mathrm{meV})$ after annealing, while the anodized area showed much larger blueshift (44 meV).

$\mathrm{Zn}$ is well known to diffuse easily at elevated temperature and has been widely used as an impurity source in impurity-induced interdiffusion [17], [18]. But our data clearly 


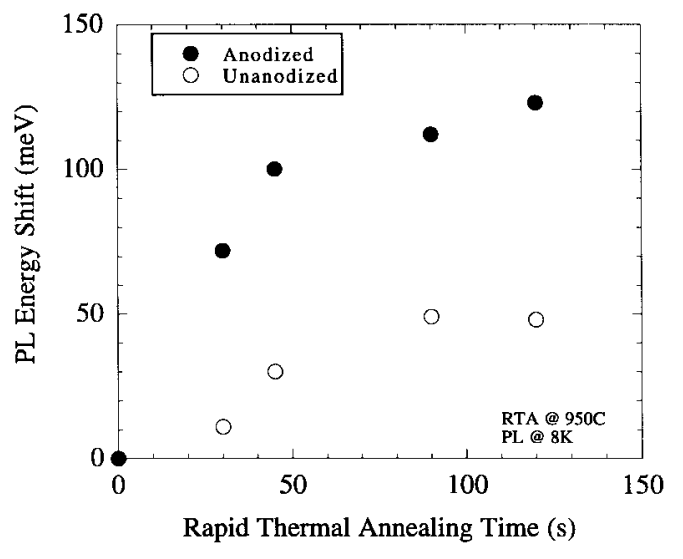

Fig. 3. PL energy shift relative to an as-grown sample against annealing time for a GaAs- $\mathrm{Al}_{0.54} \mathrm{Ga}_{0.46} \mathrm{As} \mathrm{MQW}$ structure. The sample was composed of four GaAs QW's, but for simplicity only the data for the 2.1-nm QW were shown here. The sample was annealed at $950{ }^{\circ} \mathrm{C}$ with half of the surface anodized (filled circles) and half unanodized (open circles). Similar trend held for other QW's of the structure.

demonstrated that the $\mathrm{Zn}$ dopants in the $\mathrm{p}^{+}$-GaAs substrate did not play a dominant role under these annealing conditions.

Fig. 3 shows the blueshift relative to the as-grown sample versus annealing time for a $\mathrm{QW}$ of a four QW sample. For simplicity, only the 2.1-nm-thick QW data were shown. The $\mathrm{Al}_{0.54} \mathrm{Ga}_{0.46} \mathrm{As}$ barriers were $50 \mathrm{~nm}$ thick. The GaAs top layer was $100 \mathrm{~nm}$, and the GaAs buffer layer was $500 \mathrm{~nm}$. The anodic oxide was about $60 \mathrm{~nm}$ thick, and the RTA was carried out at $950{ }^{\circ} \mathrm{C}$. For the unanodized area, the blueshift was in line with the thermal stability study of GaAs-AlGaAs QW structures [19], and went up to $50 \mathrm{meV}$ after annealing at $950{ }^{\circ} \mathrm{C}$ for $120 \mathrm{~s}$. The anodized area, however, showed much larger blueshift $\left(123 \mathrm{meV}\right.$ after annealing at $950{ }^{\circ} \mathrm{C}$ for $120 \mathrm{~s}$ ). This suggests that anodic oxide is significantly enhancing the interdiffusion. Increased energy shift due to thermal intermixing in this case when compared to Fig. 2 (5-50 meV) may be due to increased temperature (from 925 to $950^{\circ} \mathrm{C}$ ) as well as increased $\mathrm{Al}$ content in the barriers from 0.3 to 0.54 . In order to study the influence of the distance between the anodic oxide and the QW beneath it, we etched trenches into the top GaAs layer of the sample used in Fig. 2. This is shown in the insert of Fig. 4(a). The sample was first covered by thermal glue totally except an narrow stripe (window) region, and then was etched using phosphoric acid:hydrogen peroxide:de-ionized water $(1: 1: 5$ by volume $)$ at $0.5{ }^{\circ} \mathrm{C}$. After etching, the mask was removed and the process repeated to get more trenches. After etching, the whole sample was anodized except a small area for reference (denoted as "unanodized area"). The whole sample was then annealed at $950{ }^{\circ} \mathrm{C}$ for 120 s. The results were shown in Fig. 4(a). It can be seen that the unanodized area showed less blueshift compared to anodized area. And the QW beneath the trenches showed blueshift which increased with increasing trench depth, i.e., the closer the anodic oxide to the $\mathrm{QW}$, the larger blueshift. If the enhanced blueshift were due to $\mathrm{Zn}$ diffusion from the heavily Zn-doped substrate, the blueshift should not have shown noticeable dependence on the trench depth. However, further work is under way to look at the effect of substrate

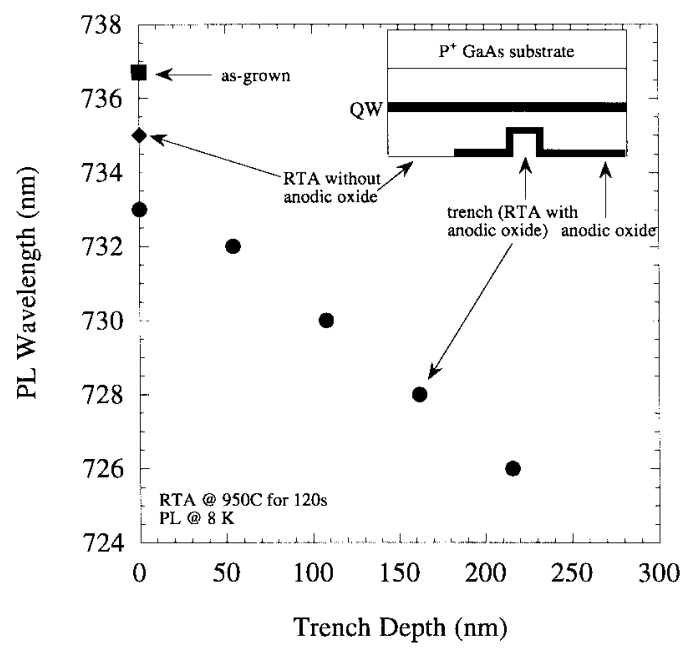

(a)

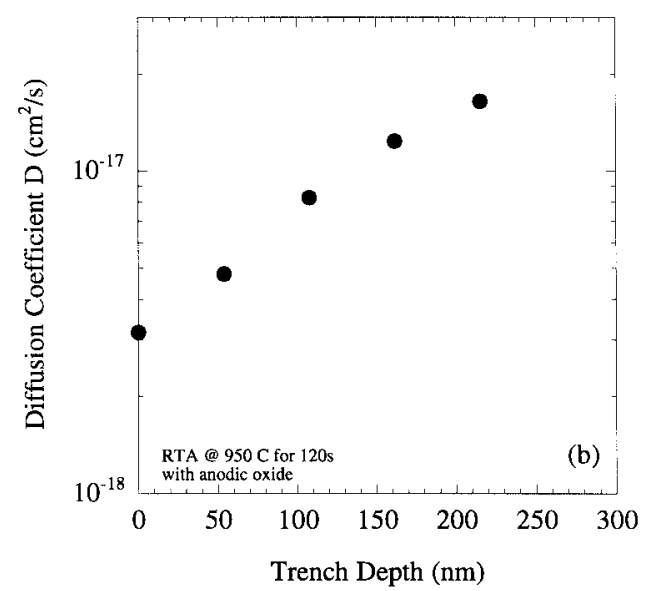

(b)

Fig. 4. (a) PL peak wavelength versus trench depth. The trench depth was measured before anodization. After the trenches with different depth were made by etching, the sample was anodized at $40 \mathrm{~mA} / \mathrm{cm}^{2}$ and then annealed at $950{ }^{\circ} \mathrm{C}$ for $120 \mathrm{~s}$. Part of the sample surface was neither etched or anodized for comparison. PL peak wavelength for this area was shown as a diamond together with the PL peak wavelength for an as-grown sample (square). (b) Diffusion coefficient versus trench depth.

doping on intermixing. The observed blueshift dependence on the trench depth could find possible applications for the fabrication of multiple wavelength laser arrays.

To determine the diffusion coefficient dependence on the trench depth, we first calculated the subband energies and wavefunctions in an intermixing modified single nonsquare QW [20], and then compared the calculated interband transition energy to the PL data. The confinement profile of this interdiffused QW was nonlinear and was modeled here by an error function. The spatially dependent electron effective mass was taken into consideration using a nonparabolic band model derived from a fourth order expansion in $k$ with the coefficients determined using a 14-band calculation, and excitonic effect was also considered. The details of the modeling was given in our previous work elsewhere [15] where the calculated Al profile after intermixing was in good agreement with measured data. Fig. 4(b) shows the resulting diffusion coefficient as a function of the trench depth. 


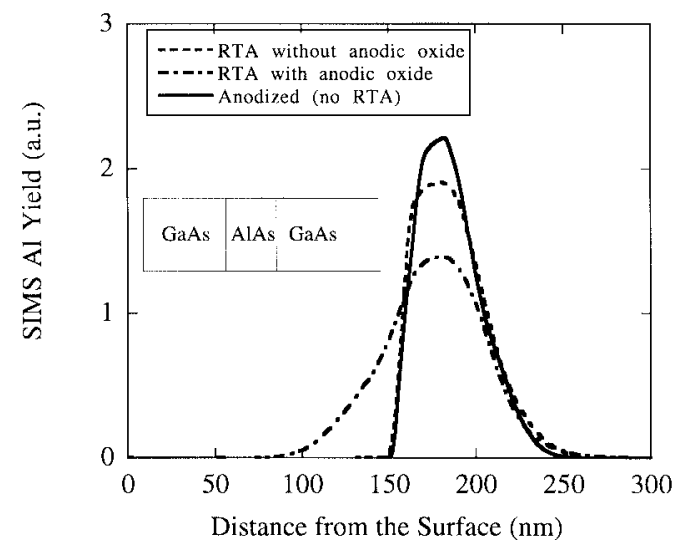

Fig. 5. Al profile from a GaAs-AlAs-GaAs substrate structure measured by SIMS. The solid line represents the Al profile from an anodized but not annealed sample. For the sample annealed at $950^{\circ} \mathrm{C}$ for $60 \mathrm{~s}$, the $\mathrm{Al}$ profiles were taken from the anodized area (dashed-dotted line) and unanodized area (dashed line).

To further confirm the effect of anodic oxide on the intermixing, we profiled Al from a GaAs-AlAs-GaAs substrate structure by secondary ion-mass spectroscopy (SIMS). The GaAs buffer layer was $1000 \mathrm{~nm}$ thick. The results were plotted in Fig. 5, showing in-depth profiles of $\mathrm{Al}$, taken under identical SIMS conditions, from an anodized, but not annealed, sample and from an annealed sample with half surface anodized and half surface unanodized. The Al profile for the anodized but not annealed sample revealed the original Al profile. The asymmetric behavior in this case is characteristic for SIMS profiles of thin buried layers and reflects the mixing induced by the ion beam used for the SIMS analysis [21], which was $10-\mathrm{keV} \mathrm{O}_{2}^{+}$beam in this work. For the unanodized area of the annealed sample, the $\mathrm{Al}$ profile was slightly broadened on both sides of the AlAs layer, presumably due to the thermal interdiffusion in the sample. For the anodized area of the annealed sample, however, the Al profile was clearly seen broadened significantly toward the surface, i.e., toward the anodic oxide, confirming the strong effect of anodic oxidation on the intermixing in GaAs-AlAs layers.

We have also applied the anodic-oxide-induced intermixing to the PL and cathodoluminescence study of GaAs-AlGaAs quantum-wire structures grown by MOCVD on V-groove patterned $\mathrm{p}^{+} \mathrm{GaAs}$ substrates. The quantum-wire structure used in this work was schematically shown in Fig. 6. The epitaxial layers consist of a GaAs buffer layer $(100 \mathrm{~nm})$, a lower $\mathrm{Al}_{0.5} \mathrm{Ga}_{0.5}$ As barrier $\left(1_{m}\right)$, a GaAs layer, an $\mathrm{Al}_{0.5} \mathrm{Ga}_{0.5} \mathrm{As}$ upper barrier $(100 \mathrm{~nm})$, finally a GaAs layer $(150 \mathrm{~nm})$. On the bottom of the V-groove, a GaAs quantum wire was formed. Cross-sectional transmission electron microscopy (TEM) was used to check the structural properties and to measure the dimension of the structure. The vertical thickness of the quantum wire at the bottom of the crescent was measured to be $9 \mathrm{~nm}$, and the sidewall QW thickness was $2.3 \mathrm{~nm}$. The first electron to heavy hole transition was evaluated [22] to be $1.558 \mathrm{eV}$ (796 $\mathrm{nm}$ ), using the vertical quantum-wire crescent thickness of 9 $\mathrm{nm}$ and a lateral potential well width of $67 \mathrm{~nm}$, and a lateral potential of $101 \mathrm{meV}$ in the conduction band and $68 \mathrm{meV}$ in the valence band. Fig. 7 showed the PL from the quantum-wire

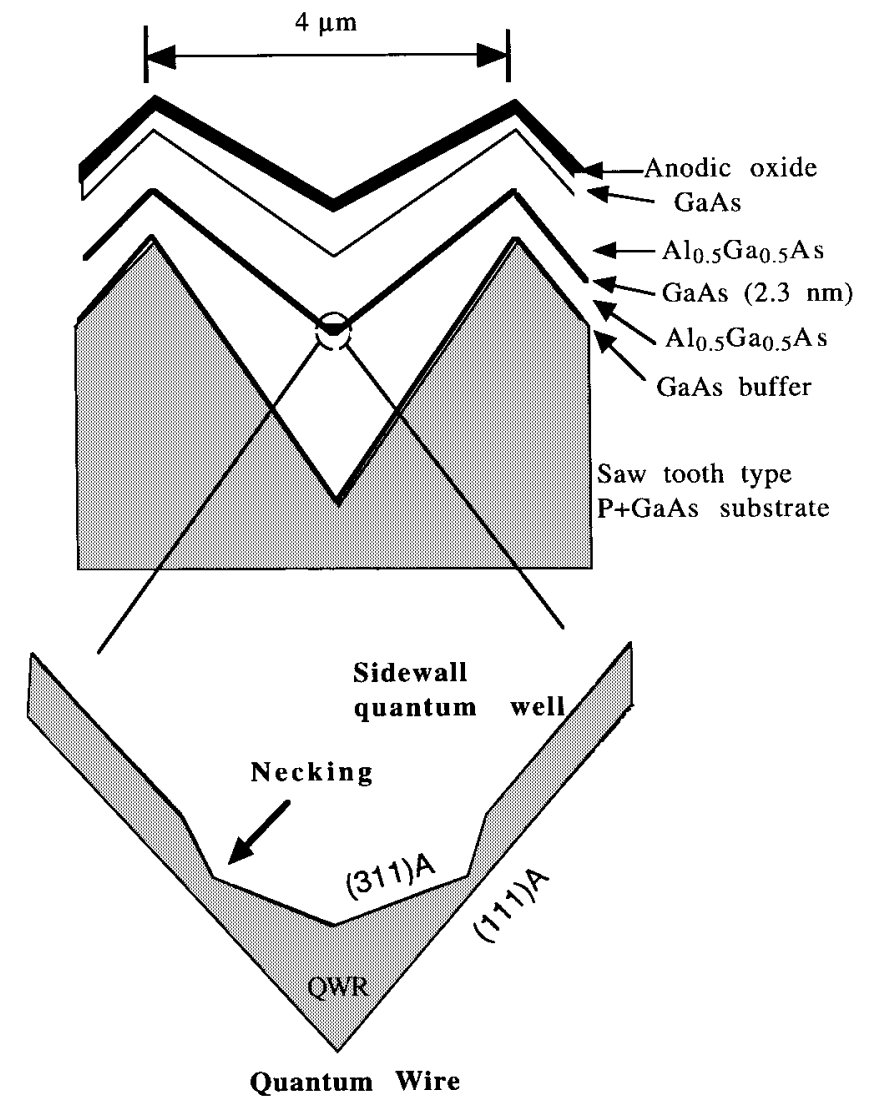

Fig. 6. Schematic diagram of the GaAs-AlGaAs quantum-wire sample used in this paper. The quantum-wire region was enlarged and shown in the lower part of the graph.

structure. No characteristic PL signal from quantum wire was seen for the as-grown sample. A hint of the quantum-wire PL was barely seen from the annealed area without anodic oxide. This is due to the fact that the volume of the quantum wire is much smaller than that of the side-wall QW's (SWQW's), so most of the photogenerated carriers were collected by the SWQW's and recombine there. The quantum-wire PL from the anodized area of the annealed sample is very clearly seen in Fig. 7. Although the annealing time was only $1 \mathrm{~s}$, it should be pointed out that the ramp time from $100{ }^{\circ} \mathrm{C}$ to $900^{\circ} \mathrm{C}$ was 16 $\mathrm{s}$, and it took a few seconds for the sample to cool down from $900{ }^{\circ} \mathrm{C}$ to below $700{ }^{\circ} \mathrm{C}$. The arrow in the graph indicates the calculated transition wavelength in the as-grown quantum wire. The assignment was also confirmed by cathodoluminescence done on similar samples annealed with anodic oxide [14]. In our earlier study, quantum-wire PL was not observed until RTA temperature was above $850^{\circ} \mathrm{C}$ from anodized sample [14], this coincides with the fact that anodic-oxideinduced intermixing in QW's emerges around $850^{\circ} \mathrm{C}$. The likely reason for the anodic-oxide enhanced quantum-wire PL signal could be that the anodic-oxide-induced intermixing in the SWQW's [23] increased the SWQW's subband energy and thus reduces the carrier confinement in the SWQW's, so more photogenerated carriers could move to the quantum wire before they recombine in the SWQW's, as the quantum wire is less affected by the anodic-oxide-induced intermixing in the vertically due to its larger thickness. 


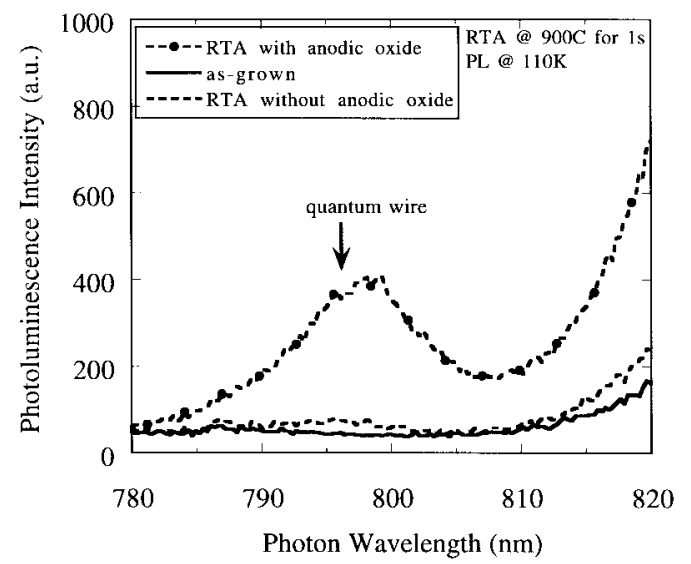

Fig. 7. PL spectra of the quantum-wire structure. The solid line was from an as-grown sample. A sample was annealed with half area anodized (dashed-dotted line) and half area unanodized (dashed line). The arrow indicates the calculated quantum-wire PL position.

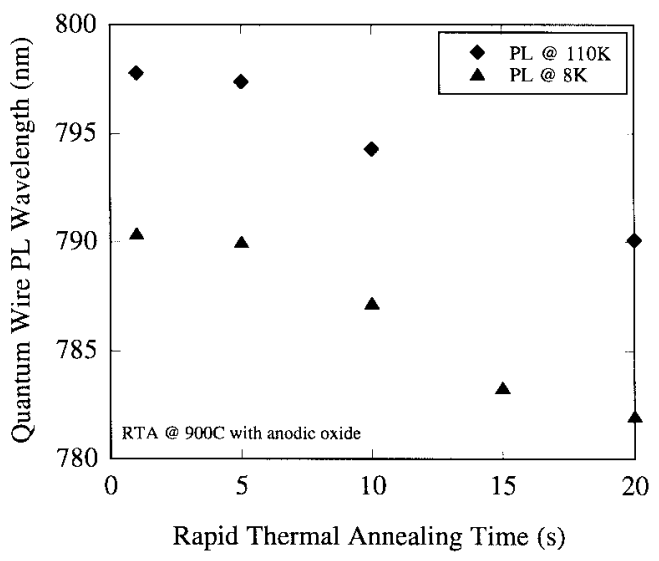

Fig. 8. Quantum-wire PL peak wavelength as a function of annealing time. The anodized samples were annealed at $900^{\circ} \mathrm{C}$. PL data were taken at 110 $\mathrm{K}$ (diamonds) and at $8 \mathrm{~K}$ (triangles).

Fig. 8 shows the quantum-wire PL peak wavelength as a function of the RTA time for samples annealed at $900{ }^{\circ} \mathrm{C}$. Two sets of data were given for the same set of samples, one for the $8 \mathrm{~K} \mathrm{PL}$ and another for the $110 \mathrm{~K} \mathrm{PL}$. It is clear that in the RTA time range, the PL from the quantum wire was blueshifted. Since no quantum-wire PL data were obtained without the anodic-oxide-induced intermixing in the SWQW's, it is difficult to say whether or not the quantumwire PL blueshift was affected significantly by the presence of the anodic oxide.

Compared to other impurity free induced intermixing techniques, anodic-oxide-induced intermixing technique is very simple and cost-effective. Increasing the output voltage of the pulser, or increasing the current density or duty cycle, or a combination of all of these parameters, we have found that the anodic oxide thickness may be increased significantly. It is expected that increased anodic oxide thickness would lead to larger blueshift. Using different electrolyte also showed encouraging results. Work is in progress to optimize the anodic-oxide-induced intermixing.
So far, the mechanism of the anodic-oxide-induced intermixing is still not very clear. It is believed that the enhanced intermixing in the anodized samples was associated with the anodic oxide, not directly to the $\mathrm{Zn}$ in the $\mathrm{Zn}$-doped $\mathrm{p}^{+}$GaAs substrates. Efforts are under way to look at the role of doping of the substrate and epilayers in anodic oxide induced intermixing. Our studies showed that the residual water in the anodic oxide plays some role, but is not the main cause [15]. The interdiffusion results suggests that the concentration of a native point defect, i.e., a group-III vacancy or interstitial, is increased at elevated temperatures when annealing under an anodic oxide. We consider it is likely that at high temperatures the oxide causes the injection of these native defects which enhance the interdiffusion. For the impurity-free enhanced interdiffusion induced in GaAs by a deposited $\mathrm{SiO}_{2}$ layer [3], it is often assumed that an increased Ga vacancy concentration enhances the interdiffusion. However, the chemical interactions between GaAs and a hydrated mixture of $\mathrm{Ga}$ and As oxides are presumably quite different than those between GaAs and $\mathrm{SiO}_{2}$ [24]. Work on the mechanism is in progress.

\section{CONCLUSION}

We have shown anodic-oxide-induced intermixing in GaAs-AlGaAs QW and quantum-wire structures. It has been demonstrated that the intermixing is significantly promoted by the presence of an anodic-oxide on the top of the structure. This effect is stronger if anodic oxide is placed closer to QW. Zn diffusion alone (without anodic oxide) induced impurity interdiffusion due to large amount of $\mathrm{Zn}$ in the heavily $\mathrm{p}^{+}$-GaAs substrates was not the cause of the anodic-oxide-induced intermixing. However, this does not rule out the possibility that interaction between $\mathrm{Zn}$ and the point defects produced by anodic oxidation may play an important role in the anodic-oxide-induced intermixing. We have applied the anodic-oxide-induced intermixing to enhance the quantum-wire PL signal in GaAs-AlGaAs quantum-wire structure grown on $\mathrm{V}$-grooved substrate.

\section{ACKNOWLEDGMENT}

The authors thank Dr. J. Marsh, Dr. A. M. Saher, and Dr. C. Largent for fruitful discussions. They also thank Dr. J. Zou for TEM measurements, P. Burke for part of the PL measurements, and Mr. M. Cohen and Dr. X. Q. Liu for experimental assistance.

\section{REFERENCES}

[1] E. H. Li, Ed., Quantum Well Intermixing for Photonics, SPIE Milestone Series 1, Bellingham, MA, 1998, vol. 145.

[2] S. F. Yu and E. H. Li, "Semiconductor lasers using diffused quantum well structures," this issue, pp. 723-735.

[3] J. H. Marsh, "Quantum well intermixing," Semiconduct. Sci. Technol., vol. 8, pp. 1136-1155, 1993.

[4] E. H. Li, Ed., Semiconductor Quantum Well Intermixing-Material Properties and Optoelectronics Applications. Amsterdam, The Netherlands: Gordon \& Breach, 1998.

[5] D. G. Deppe and N. Holonyak, Jr., "Atom diffusion and impurityinduced layer disordering in quantum well III-V semiconductor heterostructures," J. Appl. Phys., vol. 64, pp. R93-R113, 1988. 
[6] H. H. Tan, J. S. Williams, C. Jagadish, P. T. Burke, and M. Gal, "Large energy shifts in GaAs-AlGaAs quantum wells by proton irradiationinduced intermixing," Appl. Phys. Lett., vol. 68, pp. 2401-2403, 1996.

[7] I. Harrison, H. P. Ho, B. Tuck, M. Henini, and O. H. Hughes, "Zn diffusion-induced disorder in AlAs/GaAs superlattices," Semiconduct. Sci. Technol., vol. 4, pp. 841-846, 1989.

[8] S. Burkner, M. Maier, E. C. Larkins, W. Rothemound, E. P. O’Reilly, and J. D. Ralston, "Process parameter dependence of impurity-free interdiffusion in $\mathrm{GaAs} / \mathrm{AlGaAs}$ and $\mathrm{InGaAs} / \mathrm{GaAs}$ multiple quantum wells," J. Electron. Mater., vol. 24, pp. 805-812, 1995.

[9] S. G. Ayling, J. Beauvais, and J. H. Marsh, "Spatial control of quantum well intermixing in GaAs/AlGaAs using a one step process," Electron. Lett., vol. 28, pp. 2240-2241, 1992.

[10] S. Yuan, Y. Kim, C. Jagadish, P. T. Burke, M. Gal, J. Zou, D. Q. Cai, D. J. H. Cockayne, and R. M. Cohen, "Novel impurity-free interdiffusion in GaAs/AlGaAs quantum wells by anodization and rapid thermal annealing," Appl. Phys. Lett., vol. 70, pp. 1269-1271, 1997.

[11] S. K. Ghandhi, in VLSI Fabrication Principles. New York: Wiley, 1994, pp. 487-494.

[12] M. J. Grove, D. A. Hudson, P. S. Zory, R. J. Dalby, C. M. Harding, and A. Rosenberg, "Pulsed anodic oxides for III-V semiconductor device fabrication," J. Appl. Phys., vol. 76, pp. 587-589, 1994.

[13] S. Yuan, G. Li, H. H. Tan, F. Karouta, and C. Jagadish, "InGaAs GRINSCH-SQW lasers with novel carbon delta-doped contact layer," Proc. IEEE/LEOS'96, 1996, pp. 132-133.

[14] Y. Kim, S. Yuan, R. Leon, C. Jagadish, M. Gal, M. Johnston, M. Phillips, M. Kalceff, J. Zou, and D. Cockayne, "Effects of anodic oxide induced intermixing on the structural and optical properties of quantum wire structure grown on nonplanar GaAs substrate," J. Appl. Phys., vol. 80 , pp. 5014-5020, 1996.

[15] S. Yuan, Y. Kim, H. H. Tan, C. Jagadish, P. T. Burke, L. V. Dao, M. Gal, J. Zou, D. Q. Cai, D. J. H. Cockayne, and R. M. Cohen, "Interdiffusion in GaAs/AlGaAs quantum wells by pulsed anodization and subsequent rapid thermal annealing," J. Appl. Phys., vol. 83, pp. 1305-1311, Feb. 1998.

[16] C. Largent, private communication.

[17] W. D. Laidig, N. Holonyak, Jr., M. D. Camras, K. Hess, J. J. Coleman, P. D. Dapkus, and J. Bardeen, "Disorder of an AlAs/GaAs superlattice by impurity diffusion," Appl. Phys. Lett., vol. 38, pp. 776-778, 1981.

[18] Y. Nagai, K. Shigihara, S. Karakida, S. Kakimoto, M. Otsubo, and K. Ikeda, "Characteristics of laser diodes with a partially intermixed GaAs-AlGaAs quantum well," IEEE J. Quantum Electron., vol. 31, pp. $1364-1370,1995$.

[19] P. J. Hughes, E. H. Li, and B. L. Weiss, "Thermal stability of AlGaAs/GaAs single quantum well structures using photoreflectance," $J$. Vac. Sci. Technol., vol. B13, pp. 2276-2283, 1995.

[20] E. H. Li, B. L. Weiss, and K. S. Chan, "Effect of interdiffusion on the subbands in an AlGaAs/GaAs single-quantum-well structure," Phys. Rev., vol. B46, pp. 15181-15192, 1992.

[21] M. Petravic, B. G. Svensson, and J. S. Williams, "On the estimation of depth resolution during sputter profiling," Appl. Phys. Lett., vol. 62, pp. 278-280, 1993.

[22] E. Kapon, D. M. Hwang, and R. Bhat, "Stimulated emission in semiconductor quantum wire heterostructures," Phys. Rev. Lett., vol. 63, pp. 430-433, 1989.

[23] S. Yuan, Y. Kim, C. Jagadish, L. V. Dao, P. T. Burke, M. Gal, J. Zou and D. J. H. Cockayne, "Interdiffusion in GaAs/AlGaAs quantum wire structures enhanced by anodic-oxide-induced interdiffusion technique," in Proc. 8th Int. Conf. Narrow Gap Semiconductors, S. C. Shen, D. Y. Tang, G. Z. Zheng, and G. Bauer, 1998, pp. 113-118.

[24] R. M. Cohen, "Point defects and diffusion in thin films of GaAs," Mater. Sci. Eng. Rep., vol. R20, no. 4-5, pp. 167-280, 1997.

Shu Yuan (M'96) received the B.S. and M.S. degrees in physics from Fudan University, China in 1986 and 1989, respectively, and the Ph.D. degree from J. Kepler University, Austria, in 1994. His Ph.D. work was on the optical, electronic, and magneto-optical properties of IV-VI (lead salt) semiconductor structures.

He was a post-doctoral Research Associate at the University of Oklahoma from April 1994 through August 1995. He has been a Lecturer (Assistant Professor) at Nanyang Technological University, Singapore, since July 1998. His current interests are semiconductor optoelectronic materials and lasers.

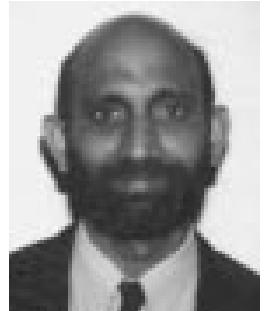

Chennupati Jagadish (S'84-M'89-SM'92) was born in Valluru Palem, India, in 1957. He received the B.Sc. degree in physics from Nagarjuna University, India, in 1977 and the M.Sc.(Tech) degree in applied physics (electronics) from Andhra University in 1980 and the M.Phil. and Ph.D degrees in semiconductor physics from University of Delhi, India, in 1982 and 1986, respectively.

$\mathrm{He}$ worked in Sri Venkateswara College, University of Delhi, as Lecturer in Electronics and Physics during 1985-1988 and moved to Queen's University, Kingston, ON, Canada, in 1988, as a Post-Doctoral Research Associate. In 1990, he moved to Australian National University where is currently a Senior Fellow and Head of the Semiconductor Growth, Characterization and Devices Group in the Department of Electronic Materials Engineering. He has given number of invited talks and served on program and advisory committees of international conferences. He has co-authored a book on "semiconducting transparent thin films" published by Institute of Physics Publishing Company and edited conference proceedings. He has published about 120 research papers in refereed journals and about 60 in refereed conference proceedings. His current research interests are in the areas of photonic devices, epitaxial growth of quantum wells, wires and dots, quantum-well intermixing, delta doping, defects in silicon and compound semiconductors, ion implantation and ultrafast photodetector materials.

Dr. Jagadish is a Fellow of the Australian Institute of Physics and a Fellow of the Institute of Physics (UK). Dr. Jagadish has been active in IEEE activities as Foundation Chair of the IEEE Australian Chapter of Electron Devices Society (EDS) and Lasers and Electro-Optics Society, Chair of the EDS Optoelectronic Devices Technical Committee, member of the EDS Publications Committee, Editor of the EDS Newsletter, Chair of the IEEE ACT section and member of the IEEE Australia Council.

Yong Kim received the Ph.D. degree in physics from Korea Advanced Institute of Science and Technology (KAIST), Seoul, Korea.

$\mathrm{He}$ is presently a senior researcher in the Semiconductor Materials Research Laboratory at the Korea Institute of Science and Technology (KIST). He was working for the growth of quantum-well structure by MOCVD. His main research interests at this time are the physics and technology of lowdimensional structure including quantum wires and dots.

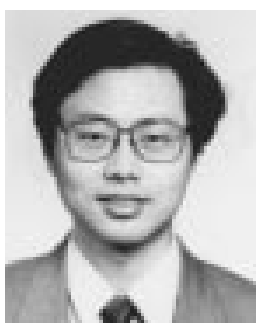

Yong Chang received the Ph.D. degree in semiconductor physics and semiconductor device physics from the National Laboratory for Infrared Physics (NLIP), Chinese Academy of Sciences, China, in 1996

$\mathrm{He}$ is with NLIP and engaged in the characterization of semiconductor materials for fabrication of infrared detectors and lasers.

Hark Hoe Tan (S'93-M'96) was born in Penang, Malaysia, in 1968. $\mathrm{He}$ received the B.E. (Elec.) and Ph.D degrees from the University of Melbourne, Australia, and the Australian National University, in 1992 and 1997, respectively.

In 1992, he joined Siemens Opto-Semiconductor, Penang, where he worked in the area of device packaging and testing. His doctoral dissertation was on ion-beam effects in GaAs-AlGaAs materials and devices. He is currently a Post-Doctoral fellow at the Australian National University. His research interests include ion implantation processing of III-V multilayer semiconductors, growth, design and fabrication of low-dimensional optoelectronic devices.

Richard M. Cohen teaches an occasional course on point defects in semiconductors in addition to his regular classes on the physics of semiconductor devices. He and his graduate students continually learn how to design useful experiments, to build appropriate test structures into epitaxial layers, and to process them in different regions of different phase diagrams.

Prof. Cohen is a member of the APS, ECS, and MRS. 


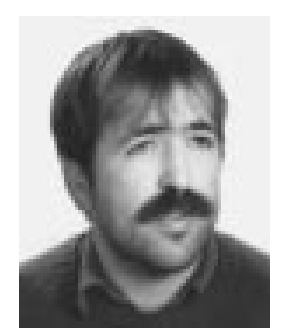

Mladen Petravic graduated from the University of Zagreb, Croatia, and received the Ph.D. degree in physics from the Australian National University.

His research interests range from the ion-beam modification and analysis of materials to the sputtering and ionization processes and desorption induced by electronic transitions.

Lap Van Dao received the Ph.D. degree from the Friedrich SchillerUniversity, Jena, Germany, in 1990. His main research interests are the development and application of ultrashort-pulse laser and laser spectroscopy.

Mike Gal (SM'95-A'96-SM'96) received the Ph.D. degree from the Eotvos Lorand University in Budapest, Hungary, in 1974.

$\mathrm{He}$ is Professor of physics at the University of New South Wales, Sydney, Australia. His research interests are the optical properties of semiconductors and optical instrumentation.

Michael C. Y. Chan was born in Hong Kong, on January 6, 1970. He received the B.Sc. (Hons.) degree in applied physics from the Hong Kong Baptist University in 1994 and the Ph.D. degree in optoelectronics from the University of Hong Kong in 1998.

His main research topics include the critical layer thickness, band structure calculation, and optical gain properties of the III-V interdiffused semiconductor quantum well. He currently conducts the III-nitride based quantum-well intermixing.
E. Herbert Li (M'84-S'87-M'88-SM'95), for photograph and biography, see this issue, p. 582.

Jeong-seok $\mathbf{O}$ was born in Korea in 1962. He received the B.S. degree in electronic engineering from Kon-Kuk University, Seoul, Korea, in 1990, and the M.S. degree in electrical and computer engineering from the University of Florida, Gainesville, in 1995, and is now working toward the Ph.D. degree in electrical and computer engineering at the University of Florida under the direction of Dr. P. S. Zory.

His research interests include modulated cap thin p-clad antiguided array lasers, quantum intersubband lasers, and $\mathrm{ZnSe}$ and $\mathrm{GaN}$ blue-green lasers."

Peter S. Zory, Jr. (M'69-SM'91-F'94) received the Ph.D. degree in physics from Carnegie-Mellon University, Pittsburgh, PA.

$\mathrm{He}$ is presently a Professor in the Electrical and Computer Engineering Department at the University of Florida, Gainesville. In previous jobs with IBM Research, McDonnell-Douglas, and General Electric, he managed all phases of diode laser development and production. His main research interests at this time are the physics and technology of quantum-well lasers. 